\title{
Solutions Without Phase-Slip for the Ginsburg-Landau Equation
}

\author{
P. Collet ${ }^{1}$ and J.-P. Eckmann ${ }^{2}$ \\ ${ }^{1}$ Centre de Physique Théorique, Ecole Polytechnique, F-91128 Palaiseau, Cedex France, \\ Laboratoire UPR A14, CNRS \\ ${ }^{2}$ Département de Physique Théorique, Université de Genève, CH-1211 Geneva 4, Switzerland
}

Received October 28, 1991

\begin{abstract}
We consider the Ginsburg-Landau equation for a complex scalar field in one dimension and consider initial data which have two different stationary solutions as their limits in space as $x \rightarrow \pm \infty$. If these solutions are not very different, then we show that the initial data will evolve to a stationary solution by a "phase melting" process which avoids "phase slips," i.e., which does not go through zero amplitude.
\end{abstract}

\section{Introduction}

In this paper, we pursue our study of the Ginsburg-Landau equation

$$
\partial_{t} u=\partial_{x}^{2} u+u-u|u|^{2}
$$

where $u: \mathbf{R} \times \mathbf{R} \rightarrow \mathbf{C}$, cf. [CE, CEE]. We shall fill in more details of the phase diagram of this equation, by studying the time evolution for initial data which are close to stationary with different amplitudes at $\pm \infty$. More precisely, define two stationary solutions $u_{ \pm}$by

$$
u_{ \pm}(x)=r_{ \pm} e^{i q_{ \pm} x+i \theta_{ \pm}},
$$

with $r_{+}=\left(1-q_{+}^{2}\right)^{1 / 2}, r_{-}=\left(1-q_{-}^{2}\right)^{1 / 2}$. Assume now that the initial data $u_{0}$ satisfy

$$
\lim _{x \rightarrow \pm \infty} u_{0}(x)-u_{ \pm}(x)=0
$$

in a sense to be described in more detail below, and assume $r_{ \pm} \approx 1$.

Under these conditions, see below for details, we shall show that the solutions have no phase slips. See Langer and Ambegaokar [LA] for an example with phase slips. Furthermore, we will show convergence to a "stationary" solution in the sense that $u(x, t)=r(x, t) e^{i \phi(x, t)}$ satisfies

$$
\sup _{x \in \mathbf{R}}\left|r^{\prime}(x, t)\right| \leqq \varepsilon, \quad \sup _{x \in \mathbf{R}}\left|\phi^{\prime \prime}(x, t)\right| \leqq \varepsilon,
$$


provided $t$ is sufficiently large. This means that on every compact interval, the solutions look for large times like a stationary solution $R(t) e^{i \Phi(t) x+i \Theta(t)}$. However, we have been unable to show that $R(t), \Phi(t)$, and $\Theta(t)$ are independent of $t$. Again, the detailed nature of this convergence will be explicit in the statement of convergence.

We change variables in (1.1), setting $u=r e^{i \phi}$, with $r, \phi: \mathbf{R} \times \mathbf{R}^{+} \rightarrow \mathbf{R}$, which leads to the equations

$$
\begin{aligned}
& \dot{r}=r^{\prime \prime}+r-r^{3}-\eta^{2} r, \\
& \dot{\eta}=\eta^{\prime \prime}+2\left(\eta \frac{r^{\prime}}{r}\right)^{\prime},
\end{aligned}
$$

with $\eta=\phi^{\prime}$, and using the notation ${ }^{\prime}=\partial_{x},{ }^{\cdot}=\partial_{t}$. Upon setting $r=1+s$, we get the system of equations in final form:

$$
\begin{aligned}
& \dot{s}=s^{\prime \prime}-2 s-3 s^{2}-s^{3}-\eta^{2}-s \eta^{2}, \\
& \dot{\eta}=\eta^{\prime \prime}+2\left(\eta \frac{s^{\prime}}{1+s}\right)^{\prime} .
\end{aligned}
$$

It is useful to define functions $F$ and $G$ and to rewrite (1.5) as

$$
\begin{aligned}
& \dot{s}=s^{\prime \prime}-2 s-2 F(s, \eta), \\
& \dot{\eta}=\eta^{\prime \prime}+G\left(s, s^{\prime}, \eta\right)^{\prime} .
\end{aligned}
$$

By the nature of the initial data, and of the time evolved solution, the right-hand side of Eq. (1.6) is 0 at $x= \pm \infty$. Since the whole problem is of diffusive type, we expect, and it will follow from the solution of the problem, that the values at infinity stay unchanged in time. However, $s$ and $\eta$ themselves are not zero at infinity, and the main purpose of this paper is to show how one deals with such a problem.

A trivial example which illustrates this mechanism is the pure diffusion equation $\dot{f}=f^{\prime \prime}$, with $\lim _{x \rightarrow-\infty} f(x, 0)=a$, $\lim _{x \rightarrow+\infty} f(x, 0)=b$. The solution $f(x, t)$ converges for every fixed, finite $x$ to $(a+b) / 2$ as $t \rightarrow \infty$, but for every fixed $t$ the limits at infinity are still $a$, respectively $b$, see also the explicit example at the end of the paper.

Our method consists in introducing a new independent variable $z: \mathbf{R} \times \mathbf{R}^{+} \rightarrow \mathbf{R}$ which vanishes at $\infty$ (s does not), and so we define

where $F$ is the polynomial

$$
z=s+F(s, \eta)
$$

$$
2 F(s, \eta)=3 s^{2}+s^{3}+\eta^{2}+s \eta^{2}
$$

However, our proof uses a little less: We only need to know that $F(\varepsilon x, \varepsilon y)=\mathcal{O}\left(\varepsilon^{2}\right)$ as $\varepsilon \rightarrow 0$, where $x, y \in \mathbf{R}$, and that $F$ is $\mathscr{C}^{2}$. Clearly, there is a function $f$ such that for sufficiently small $z$ and $\eta$ we have

$$
s=z+f(z, \eta)
$$

Note that $f(\varepsilon z, \varepsilon \eta)=\mathcal{O}\left(\varepsilon^{2}\right)$. We will now change variables in (1.5) from $s$ to $z$. Denoting partial derivatives with appended subscripts, we have

$$
\begin{aligned}
s^{\prime} & =\left(1+f_{z}\right) z^{\prime}+f_{\eta} \eta^{\prime}, \\
s^{\prime \prime} & =\left(1+f_{z}\right) z^{\prime \prime}+f_{z z} z^{2}+2 f_{z \eta} z^{\prime} \eta^{\prime}+f_{\eta \eta} \eta^{\prime 2}+f_{\eta} \eta^{\prime \prime} .
\end{aligned}
$$


We introduce notation to simplify the sums of derivatives. $D_{1}, D_{2}$ denote the vectors

$$
\begin{aligned}
& D_{1}=\left(z^{\prime}, \eta^{\prime}, 0,0,0\right), \\
& D_{2}=\left(z^{\prime \prime}, \eta^{\prime \prime}, z^{\prime 2}, z^{\prime} \eta^{\prime}, \eta^{2}\right),
\end{aligned}
$$

so that (1.7) becomes

$$
\begin{gathered}
s^{\prime}=z^{\prime}+H_{1} D_{1}, \\
s^{\prime \prime}=z^{\prime \prime}+H_{2} D_{2},
\end{gathered}
$$

with

$$
\begin{aligned}
& H_{1}=\left(f_{z}, f_{\eta}, 0,0,0\right), \\
& H_{2}=\left(f_{z}, f_{\eta}, f_{z z}, 2 f_{z \eta}, f_{\eta \eta}\right),
\end{aligned}
$$

and $H_{i} D_{k}$ denoting the scalar product. Note that if $z$ and $\eta$ are small in $L^{\infty}$ then $H_{1}$ and the first two components of $H_{2}$ are small in $L^{\infty}$. Furthermore, the last three components of $\mathrm{H}_{2}$ are bounded. We call $\mathscr{H}$ the set of functions like $\mathrm{H}_{1}, \mathrm{H}_{2}$ :

$$
\begin{aligned}
\mathscr{H}= & \left\{h_{1}, \ldots, h_{5}: \mathbf{R} \times \mathbf{R}^{+} \rightarrow \mathbf{R} \mid\left\|h_{i}(\varepsilon f, \varepsilon g)\right\|_{\infty} \leqq \mathcal{O}(\varepsilon), \quad i=1,2\right. \\
& \left.\left\|h_{j}(\varepsilon f, \varepsilon g)\right\|_{\infty} \leqq \mathcal{O}(1), j=3,4,5, \text { when }\|f\|_{\infty},\|g\|_{\infty} \leqq 1\right\} .
\end{aligned}
$$

Consider next the term $G$ in the second equation of (1.6). We define $H_{0}=f(z, \eta)$. Then

$$
g\left(z, z^{\prime}, \eta\right) \equiv G\left(s, s^{\prime}, \eta\right)=G\left(z+H_{0}, z^{\prime}+H_{1} D_{1}, \eta\right)
$$

and therefore

$$
\frac{d}{d x} G\left(s, s^{\prime}, \eta\right)=\left(z^{\prime}+H_{1} D_{1}\right) G_{s}+\left(z^{\prime \prime}+H_{2} D_{2}\right) G_{s^{\prime}}+\eta^{\prime} G_{\eta} .
$$

Here, all $G$ 's are evaluated at $\left(z+H_{0}, z^{\prime}+H_{1} D_{1}, \eta\right)$. Recalling the definition of $G$ in (1.5), (1.6), we see that

$$
\frac{d}{d x} G\left(s, s^{\prime}, \eta\right)=H^{(G)} D_{2}
$$

We can now transform the system (1.5) as follows:

$$
\begin{aligned}
\dot{z} & =\left(1+F_{s}\right) \dot{s}+F_{\eta} \dot{\eta} \\
& =\left(1+F_{s}\right)\left(\left(z^{\prime \prime}+H_{2} D_{2}\right)-2 z\right)+F_{\eta}\left(\eta^{\prime \prime}+H^{(G)} D_{2}\right) \\
& =z^{\prime \prime}-2 A z+H^{(z)} D_{2},
\end{aligned}
$$

where $A=\left(1+F_{s}\right)$ is a function of $z$ and $\eta$ which satisfies, for $\|\eta\|_{\infty},\|z\|_{\infty} \leqq \varepsilon$,

$$
A=1+\mathcal{O}(\varepsilon) \text {. }
$$

The equation for $\eta$ is immediate from (1.8) and we get the system of equations

$$
\begin{aligned}
& \dot{z}=z^{\prime \prime}-2 A z+H^{(z)} D_{2}, \\
& \dot{\eta}=\eta^{\prime \prime}+H^{(G)} D_{2},
\end{aligned}
$$

with $H^{(z)}, H^{(G)}$ in $\mathscr{H}$ and $A-1=\mathcal{O}(\varepsilon)$.

We now consider in more detail the second equation of (1.10). We encounter here a problem which is similar to the one found in the relation between $s$ and $z$. 
Namely, since the limits of $\eta$ at $\infty$ and $-\infty$ are not necessarily the same, we do not expect $\eta \in L^{2}$, which would be useful in view of the application of "energy methods."

But we can now use the fact that the limits at infinity stay constant in time and express $\eta$ as an approximate solution $\zeta$ which has the correct behavior at infinity and then bound the difference $y=\eta-\zeta$ in $L^{2}$.

We first recall that $s=z+f(z, \eta)$, and therefore the right-hand side of the second equation of (1.6) can be written as

$$
\begin{aligned}
\eta^{\prime \prime} & +\left(\frac{z^{\prime}+f_{\eta}(z, \eta) \eta^{\prime}+f_{z}(z, \eta) z^{\prime}}{1+z+f(z, \eta)} \eta\right)^{\prime} \\
& \equiv \eta^{\prime \prime}+\left(\eta \eta^{\prime} Q_{1}(z, \eta)\right)^{\prime}+\left(\eta z^{\prime} Q_{2}(z, \eta)\right)^{\prime}
\end{aligned}
$$

where

$$
\begin{array}{ll}
Q_{1}=f_{\eta}(1+z+f)^{-1}, & Q_{1}=\mathcal{O}(\varepsilon) \\
Q_{2}=\left(1+f_{z}\right)(1+z+f)^{-1}, & Q_{2}=\mathcal{O}(1) .
\end{array}
$$

With this notation, we have

$$
\dot{\eta}=\eta^{\prime \prime}+\left(\eta \eta^{\prime} Q_{1}\right)^{\prime}+\left(\eta z^{\prime} Q_{2}\right)^{\prime}
$$

We define the approximate quantity $\zeta$ as the solution of

$$
\dot{\zeta}=\zeta^{\prime \prime}+\left(\eta \zeta^{\prime} Q_{1}\right)^{\prime},
$$

with the boundary condition $\zeta( \pm \infty, t=0)=\eta( \pm \infty, t=0)$. This equation is of the type

$$
\dot{\zeta}=\partial_{x}\left(1+\eta Q_{1}\right) \partial_{x} \zeta
$$

We define $y=\eta-\zeta$, and the equation for $y$ is

$$
\dot{y}=y^{\prime \prime}+\left(\eta y^{\prime} Q_{1}\right)^{\prime}+\left(\eta z^{\prime} Q_{2}\right)^{\prime} .
$$

We now formulate some assumptions on $z, \eta, \zeta$, and $y$, and we shall show that if these assumptions hold at time $t=0$, then they hold for all $t \geqq 0$.

Definition. We denote by $\mathscr{B}_{\varepsilon}$ the set of all functions $z, \eta, \zeta$, and $y$, mapping $\mathbf{R} \rightarrow \mathbf{R}$, which satisfy

$$
\max \int_{-\infty}^{\infty} d x\left(z^{2}+z^{\prime 2}+z^{\prime \prime 2}+\eta^{\prime 2}+\eta^{\prime \prime 2}+y^{2}+y^{\prime 2}\right)+\|\zeta\|_{\infty}^{2} \leqq \varepsilon^{2} .
$$

Main Assumption. The initial data of the problem (1.1)-or equivalently, of (1.6), (1.10), (1.12), (1.14)—with $\zeta(t=0, \cdot)=\eta(t=0, \cdot)$ are in $\mathscr{B}_{\varepsilon}$.

Our result is

Theorem 1.1. There is an $\varepsilon_{0}>0$ such that if the initial data satisfy the Main Assumption with $\varepsilon<\varepsilon_{0}$, then the solution is, for all $t \geqq 0$, in $\mathscr{B}_{\varepsilon}$.

Remark. The conditions in the Main Assumption are somewhat redundant, since, e.g., the bound on $z^{\prime}$ follows from that on $z, z^{\prime \prime}$, and $y, \zeta$, and $\eta$ are not independent variables. For the sake of clarity we keep all the conditions. 


\section{Proof of Theorem 1.1}

Before going into details we recall that $z$ and $\eta$ are real functions, and we will use the shorthand $\int f=\int_{-\infty}^{\infty} d x f(x)$. Furthermore, we shall throughout make $\varepsilon_{0}$ smaller as the need occurs.

We denote by $\xi$ the set of functions $z, \eta, \zeta$, and $y$. If $\xi \in \mathscr{B}_{\varepsilon}$ then we have in particular

$$
\begin{array}{r}
\|z\|_{2},\left\|z^{\prime}\right\|_{2},\left\|z^{\prime \prime}\right\|_{2} \leqq \varepsilon \\
\left\|\eta^{\prime}\right\|_{2},\left\|\eta^{\prime \prime}\right\|_{2} \leqq \varepsilon \\
\|y\|_{2},\left\|y^{\prime}\right\|_{2},\|\zeta\|_{\infty} \leqq \varepsilon
\end{array}
$$

We shall need repeatedly a set of simple inequalities, which we call A, B... Inequality $A$.

$$
\left\|f^{\prime 2}\right\|_{2}=\left\|f^{\prime}\right\|_{4}^{2} \leqq 3\|f\|_{\infty}\left\|f^{\prime \prime}\right\|_{2} .
$$

Inequality $B_{\mu}$. If $a, b>0$ then, for all $\mu>0$,

$$
a \cdot b \leqq \frac{1}{2}\left(\mu a^{2}+\frac{1}{\mu} b^{2}\right) .
$$

Inequality $C_{\mu}$. For all $\mu>0$,

$$
\|f \cdot g\|_{1} \leqq \frac{1}{2}\left(\mu\|f\|_{2}^{2}+\frac{1}{\mu}\|g\|_{2}^{2}\right) .
$$

Inequality $D$ (Sobolev inequality).

$$
\|f\|_{\infty}^{2} \leqq\|f\|_{2}\left\|f^{\prime}\right\|_{2}
$$

Proofs. The proofs are almost trivial. To prove A, we observe that

$$
0 \leqq\left\|f^{\prime 2}\right\|_{2}^{2}=\int f^{\prime 4}=\int f^{\prime} f^{\prime 3}=-3 \int f f^{\prime 2} f^{\prime \prime} \leqq 3\|f\|_{\infty}\left\|f^{\prime 2}\right\|_{2}\left\|f^{\prime \prime}\right\|_{2},
$$

from which the assertion follows at once. Clearly, B, C, D are trivial.

The inequalities (2.1) imply the further inequalities

$$
\begin{aligned}
\|z\|_{\infty},\left\|z^{\prime}\right\|_{\infty} & \leqq \varepsilon \\
\|\eta\|_{\infty},\left\|\eta^{\prime}\right\|_{\infty} & \leqq 4 \varepsilon, \\
\|y\|_{\infty} & \leqq 3 \varepsilon .
\end{aligned}
$$

Indeed, the bounds on $z, z^{\prime}, \eta^{\prime}$ follow from (2.1) by the Sobolev inequality. The bound on $\|y\|_{\infty}$ follows from Inequality $\mathrm{A}$ by

$$
\|y\|_{\infty}^{2}=2\left|\int y y^{\prime}\right| \leqq 3\left\|y^{\prime}\right\|_{2}\|y\|_{2} \leqq 3 \varepsilon^{2},
$$

and the bound on $\|\eta\|_{\infty}$ now follows from $\eta=y+\zeta$.

After these preliminary estimates, we can now bound the various quantities in (1.15). We begin by bounding $L=\frac{1}{2} \int z^{\prime 2}+\frac{1}{2} \int \eta^{\prime 2}$. We shall show $\dot{L}<0$ and in fact 
even a little bit more. Integrating by parts, we see from (1.10) that

$$
\begin{aligned}
\dot{L}= & -\int z^{\prime \prime} \dot{z}-\int \eta^{\prime \prime} \dot{\eta} \\
= & -\int z^{\prime \prime 2}-2 A \int z^{2}-\int H^{(z)} z^{\prime \prime} D_{2} \\
& -\int \eta^{\prime \prime 2}-\int H^{(G)} \eta^{\prime \prime} D_{2} .
\end{aligned}
$$

Since $H^{(z)}, H^{(G)} \in \mathscr{H}$, we shall bound their components in $L^{\infty}$, and bound then the integrals of the terms occurring in $z^{\prime \prime} D_{2}$ and $\eta^{\prime \prime} D_{2}$.

There are 9 different terms in $z^{\prime \prime} D_{2}$ and $\eta^{\prime \prime} D_{2}$, most of which are trivially bounded. To be precise, we bound $\int z^{\prime \prime 2}$ and $\int \eta^{\prime \prime 2}$ by themselves, (and since they have small coefficients they will be dominated by the explicit integrals in (2.4)). Similarly, by Inequality $C_{1}$, we have $\int\left|z^{\prime \prime} \eta^{\prime \prime}\right| \leqq \frac{1}{2} \int\left(z^{\prime \prime 2}+\eta^{\prime \prime 2}\right)$. Using Inequality $\mathrm{A}$, the term $\int\left|\eta^{\prime \prime} z^{\prime 2}\right|$ is bounded as follows:

$$
\begin{aligned}
\int\left|\eta^{\prime \prime} z^{\prime 2}\right| & \leqq\left\|\eta^{\prime \prime}\right\|_{2}\left\|z^{\prime 2}\right\|_{2} \leqq \varepsilon\left\|\eta^{\prime \prime}\right\|_{2}^{2}+\varepsilon^{-1}\left\|z^{\prime 2}\right\|_{2}^{2} \\
& \leqq \varepsilon\left\|\eta^{\prime \prime}\right\|_{2}^{2}+9 \varepsilon^{-1}\|z\|_{\infty}^{2}\left\|z^{\prime \prime}\right\|_{2}^{2}=\mathcal{O}(\varepsilon) \int\left(z^{\prime \prime 2}+\eta^{\prime \prime 2}\right) .
\end{aligned}
$$

The same method leads to the bounds

$$
\begin{aligned}
& \int\left|z^{\prime \prime} z^{\prime 2}\right| \leqq \mathcal{O}(\varepsilon) \int z^{\prime \prime 2} \\
& \int\left|\eta^{\prime \prime} \eta^{\prime 2}\right| \leqq \mathcal{O}(\varepsilon) \int \eta^{\prime \prime 2} \\
& \int\left|z^{\prime \prime} \eta^{\prime 2}\right| \leqq \mathcal{O}(\varepsilon) \int\left(z^{\prime \prime 2}+\eta^{\prime \prime 2}\right) .
\end{aligned}
$$

Note next that, by Inequality $B_{1}$,

$$
\left|z^{\prime} \eta^{\prime}\right| \leqq \frac{1}{2}\left(\left|z^{\prime}\right|^{2}+\left|\eta^{\prime}\right|^{2}\right)
$$

Therefore,

$$
\begin{aligned}
& \int\left|z^{\prime \prime} z^{\prime} \eta^{\prime}\right| \leqq \mathcal{O}(\varepsilon) \int\left(z^{\prime \prime 2}+\eta^{\prime 2}\right), \\
& \int\left|\eta^{\prime \prime} \eta^{\prime} z^{\prime}\right| \leqq \mathcal{O}(\varepsilon) \int\left(z^{\prime \prime 2}+\eta^{\prime \prime 2}\right) .
\end{aligned}
$$

Adding all terms together, and noting that $H^{(z)}, H^{(G)} \in \mathscr{H}$, we find that

$$
\dot{L}=\frac{d}{d t}\left(\frac{1}{2} \int z^{\prime 2}+\frac{1}{2} \int \eta^{\prime 2}\right) \leqq-(1-\mathcal{O}(\varepsilon))\left[\int z^{\prime \prime 2}+2 \int z^{\prime 2}+\int \eta^{\prime \prime 2}\right] .
$$

We next consider the quantity $W=\frac{1}{2} \int z^{2}$. We get from Eq. (1.10),

$$
\dot{W}=\int z \dot{z}=\int z z^{\prime \prime}-2 \int A z^{2}+\int z H^{(z)} D_{2}=-\int z^{\prime 2}-2 \int A z^{2}+\int z H^{(z)} D_{2} .
$$

The terms coming from $z H^{(z)} D_{2}$ are bounded as follows:

$$
\begin{aligned}
\int\left|z z^{\prime \prime}\right| & \leqq\|z\|_{2}\left\|z^{\prime \prime}\right\|_{2} \leqq\|z\|_{2}^{2}+\left\|z^{\prime \prime}\right\|_{2}^{2}, \\
\int\left|z \eta^{\prime \prime}\right| & \leqq\|z\|_{2}\left\|\eta^{\prime \prime}\right\|_{2} \leqq\|z\|_{2}^{2}+\left\|\eta^{\prime \prime}\right\|_{2}^{2}, \\
\int\left|z z^{\prime 2}\right| & \leqq \varepsilon\|z\|_{2}^{2}+\varepsilon^{-1} \int z^{\prime 4} \leqq \varepsilon\|z\|_{2}^{2}+9 \varepsilon^{-1}\left\|z^{\prime \prime}\right\|_{2}^{2}\|z\|_{\infty}^{2} \leqq \mathcal{O}(\varepsilon)\left(\|z\|_{2}^{2}+\left\|z^{\prime \prime}\right\|_{2}^{2}\right), \\
\int\left|z \eta^{\prime 2}\right| & \leqq \mathcal{O}(\varepsilon)\left(\|z\|_{2}^{2}+\left\|\eta^{\prime \prime}\right\|_{2}^{2}\right), \\
\int\left|z z^{\prime} \eta^{\prime}\right| & \leqq \mathcal{O}(\varepsilon)\left(\|z\|_{2}^{2}+\left\|\eta^{\prime \prime}\right\|_{2}^{2}+\left\|z^{\prime \prime}\right\|_{2}^{2}\right) .
\end{aligned}
$$

Since the first two terms are multiplied by terms of order $\varepsilon$, and the last three by terms of order 1 , we find

$$
\dot{W}=\frac{1}{2} \frac{d}{d t} \int z^{2} \leqq-(1-\mathcal{O}(\varepsilon)) \int\left(z^{\prime 2}+2 z^{2}\right)+\mathcal{O}(\varepsilon) \int\left(z^{\prime \prime 2}+\eta^{\prime \prime 2}\right) .
$$


We next provide bounds on the second derivatives. We let $V=\frac{1}{2} \int\left(z^{\prime \prime 2}+\eta^{\prime \prime 2}\right)$. We find

$$
\frac{1}{2} \frac{d}{d t} \int z^{\prime \prime 2}=\int z^{(i v)} \dot{z}=\int z^{(i v)} z^{\prime \prime}-2 \int A z^{(i v)} z+\int H^{(z)} z^{(i v)} D_{2} .
$$

We again bound the various terms, but the calculation is somewhat complicated by the fact that we now need to integrate by parts and that, e.g., the coefficient $A$ is not constant. Furthermore, the estimates are somewhat asymmetric in the handling of $z$ and $\eta$ because we cannot use $\left\|\eta^{\prime}\right\|_{2}$ in the upper bounds (because there will be no compensating negative term).

The first term leads to $\int z^{(i v)} z^{\prime \prime} \leqq-\int z^{\prime \prime \prime 2}$, and this term will be responsible for the success of the final bound. We deal next in much detail with the term $\int h(z, \eta) z^{(i v)} z$, where $h=A-1$ is a small function, with bounded partial derivatives. Then

$$
\int h(z, \eta) z^{(i v)} z=-\int h z^{\prime \prime \prime} z^{\prime}-\int h_{z} z^{\prime \prime \prime} z^{\prime} z-\int h_{\eta} z^{\prime \prime \prime} \eta^{\prime} z
$$

Note that $\|h\|_{\infty}=\mathcal{O}(\varepsilon)$, and $\left\|h_{z}\right\|_{\infty},\left\|h_{\eta}\right\|_{\infty}=\mathcal{O}(1)$. Taking absolute values we get the bound

$$
\begin{aligned}
\left|\int h z^{(i v)} z\right| \leqq & \mathcal{O}(\varepsilon)\left(\left\|z^{\prime \prime \prime}\right\|_{2}^{2}+\left\|z^{\prime}\right\|_{2}^{2}\right)+\mathcal{O}(1)\left(\varepsilon\left\|z^{\prime \prime \prime}\right\|_{2}^{2}+\varepsilon^{-1}\left\|z^{\prime}\right\|_{2}^{2}\|z\|_{\infty}^{2}\right) \\
& +\mathcal{O}(\varepsilon)\left(\varepsilon\left\|z^{\prime \prime \prime}\right\|_{2}^{2}+\varepsilon^{-1}\left\|\eta^{\prime}\right\|_{\infty}^{2}\|z\|_{2}^{2}\right) \\
\leqq & \mathcal{O}(\varepsilon)\left(\left\|z^{\prime \prime \prime}\right\|_{2}^{2}+\|z\|_{2}^{2}+\left\|z^{\prime}\right\|_{2}^{2}\right) .
\end{aligned}
$$

We next consider the terms which come from $\int H^{(z)} z^{(i v)} D_{2}$. We begin with $\int h z^{(i v)} z^{\prime 2}$. Of course, $h$ does not denote the same function as above, and it obeys now a bound $\mathcal{O}(1)$. We can again expand and integrate by parts as before

$$
\int h z^{(i v)} z^{\prime 2}=-2 \int h z^{\prime \prime \prime} z^{\prime \prime} z^{\prime}-\int h_{z} z^{\prime \prime \prime} z^{\prime 3}-\int h_{\eta} z^{\prime \prime \prime} z^{\prime 2} \eta^{\prime}
$$

We bound each of the terms as follows:

$$
\begin{aligned}
\int\left|h z^{\prime \prime \prime} z^{\prime \prime} z^{\prime}\right| & \leqq\left\|h z^{\prime}\right\|_{\infty} \int\left(z^{\prime \prime \prime 2}+z^{\prime \prime 2}\right), \\
\int\left|h_{z} z^{\prime \prime \prime} z^{\prime 3}\right| & \leqq\left\|h_{z} z^{\prime}\right\|_{\infty}\left(\varepsilon \int z^{\prime \prime \prime 2}+\varepsilon^{-1} \int z^{\prime 4}\right) \leqq \mathcal{O}(\varepsilon)\left\|h_{z} z^{\prime}\right\|_{\infty} \int\left(z^{\prime \prime \prime 2}+z^{\prime \prime 2}\right), \\
\int\left|h_{\eta} z^{\prime \prime \prime} z^{\prime 2} \eta^{\prime}\right| & \leqq h_{\eta} \eta^{\prime}\left\|_{\infty}\left(\varepsilon \int z^{\prime \prime \prime 2}+\varepsilon^{-1} \int z^{\prime 4}\right) \leqq \mathcal{O}(\varepsilon)\right\| h_{\eta} \eta^{\prime} \|_{\infty} \int\left(z^{\prime \prime \prime 2}+z^{\prime \prime 2}\right) .
\end{aligned}
$$

Similarly,

$$
\int h z^{(i v)} \eta^{\prime 2}=-2 \int h z^{\prime \prime \prime} \eta^{\prime \prime} \eta^{\prime}-\int h_{\eta} z^{\prime \prime \prime} \eta^{\prime 3}-\int h_{z} z^{\prime \prime \prime} \eta^{\prime 2} z^{\prime},
$$

and this leads to bounds,

$$
\begin{aligned}
\int\left|h z^{\prime \prime \prime} \eta^{\prime \prime} \eta^{\prime}\right| & \leqq\left\|h \eta^{\prime}\right\|_{\infty} \int\left(z^{\prime \prime \prime 2}+\eta^{\prime \prime 2}\right), \\
\int\left|h_{\eta} z^{\prime \prime \prime} \eta^{\prime 3}\right| & \leqq \mathcal{O}(\varepsilon)\left\|h_{\eta} \eta^{\prime}\right\|_{\infty} \int\left(z^{\prime \prime \prime 2}+\eta^{\prime \prime 2}\right), \\
\int\left|h_{z} z^{\prime \prime \prime} z^{\prime} \eta^{\prime 2}\right| & \leqq \mathcal{O}(\varepsilon)\left\|h_{z} z^{\prime}\right\|_{\infty} \int\left(z^{\prime \prime \prime 2}+\eta^{\prime \prime 2}\right) .
\end{aligned}
$$

We next bound $\left|\int h z^{(i v)} z^{\prime} \eta^{\prime}\right|$ by $\left|\int h z^{(i v)}\left(z^{\prime 2}+\eta^{\prime 2}\right)\right|$ which we just handled. The term $\int h z^{(i v)} \eta^{\prime \prime}$ is rewritten as

$$
\int h z^{(i v)} \eta^{\prime \prime} \doteq-2 \int h z^{\prime \prime \prime} \eta^{\prime \prime \prime}-\int h_{z} z^{\prime \prime \prime} \eta^{\prime \prime} z^{\prime}-\int h_{\eta} z^{\prime \prime \prime} \eta^{\prime \prime} \eta^{\prime},
$$

and this can be bounded by

$$
\mathcal{O}(\varepsilon)\left(\left\|z^{\prime \prime \prime}\right\|_{2}^{2}+\left\|\eta^{\prime \prime \prime}\right\|_{2}^{2}+\left\|z^{\prime \prime}\right\|_{2}^{2}+\left\|\eta^{\prime \prime}\right\|_{2}^{2}\right)
$$

This completes the analysis of $\int z^{\prime \prime 2}$. 
The equation for $\int \eta^{\prime 2}$ is

$$
\frac{1}{2} \frac{d}{d t} \int \eta^{\prime \prime 2}=\int \eta^{(i v)} \dot{\eta}=\int \eta^{(i v)} \eta^{\prime \prime}+\int \eta^{(i v)} H^{(G)} D_{2}
$$

The roles of $\eta$ and $z$ are now exchanged, with the quantity $\int H^{(G)} \eta^{(i v)} D_{2}$ replacing $\int H^{(z)} z^{(i v)} D_{2}$. Therefore, this term is again bounded by an expression of the form of (2.8). Adding up all the terms, we finally get that

$$
\dot{V}=\frac{1}{2} \frac{d}{d t} \int\left(z^{\prime \prime 2}+\eta^{\prime \prime 2}\right) \leqq-(1-\mathcal{O}(\varepsilon)) \int\left(z^{\prime \prime \prime 2}+\eta^{\prime \prime \prime 2}\right)+\mathcal{O}(\varepsilon) \int\left(z^{\prime \prime 2}+z^{\prime 2}+\eta^{\prime \prime 2}\right) .
$$

We next want to bound the $L^{2}$ norm of $y$, and we consider therefore $Z=\frac{1}{2} \int y^{2}$. We have, cf. Eq. (1.14),

$$
\dot{Z}=\int y \dot{y}=-\int y^{\prime 2}-\int \eta y^{\prime 2} Q_{1}-\int y^{\prime} \eta Q_{2} z^{\prime} .
$$

The sum of the first two terms on the right-hand side of (2.10) is bounded above by $-(1-\partial(\varepsilon)) \int y^{\prime 2}$, if $\varepsilon$ is sufficiently small. The last term is bounded by

$$
\mathcal{O}(1)\left(\varepsilon \int y^{\prime 2}+\varepsilon^{-1} \int z^{\prime 2}\left|\eta Q_{2}\right|^{2}\right) \text {. }
$$

The first term in (2.11) is again absorbed easily in $\int y^{\prime 2}$, whereas the second is bounded by

so that we find

$$
\mathcal{O}\left(\varepsilon^{-1}\right)\left\|z^{\prime}\right\|_{2}^{2}\left\|\eta Q_{2}\right\|_{\infty}^{2}
$$

$$
\dot{Z} \leqq-(1-\mathcal{O}(\varepsilon)) \int y^{\prime 2}+\mathcal{O}(\varepsilon) \int z^{\prime 2} .
$$

We finally bound $y^{\prime}$ in $L^{2}$. By Eq. (1.14), we get, for $M=\frac{1}{2} \int y^{\prime 2}$,

$$
\begin{aligned}
\dot{M} & =-\int y^{\prime \prime 2}-\int y^{\prime \prime}\left(\eta y^{\prime} Q_{1}\right)^{\prime}-\int y^{\prime \prime}\left(\eta z^{\prime} Q_{2}\right)^{\prime} \\
& \leqq-(1-\mathcal{O}(\varepsilon)) \int y^{\prime \prime 2}-\int y^{\prime \prime} y^{\prime}\left(\eta Q_{1}\right)^{\prime}-\int y^{\prime \prime} z^{\prime \prime}\left(\eta Q_{2}\right)-\int y^{\prime \prime} z^{\prime}\left(\eta Q_{2}\right)^{\prime} .
\end{aligned}
$$

Consider first the term $\int y^{\prime \prime} y^{\prime}\left(\eta Q_{1}\right)^{\prime}$. It can be rewritten as

$$
\int y^{\prime \prime} y^{\prime} \eta^{\prime} Q_{1}+\int y^{\prime \prime} y^{\prime} \eta^{\prime} \eta\left(Q_{1}\right)_{\eta}+\int y^{\prime \prime} y^{\prime} z^{\prime} \eta\left(Q_{1}\right)_{z},
$$

with $\left\|Q_{1}\right\|_{\infty},\left\|\eta\left(Q_{1}\right)_{\eta}\right\|_{\infty},\left\|\eta\left(Q_{1}\right)_{z}\right\|_{\infty}=\mathcal{O}(\varepsilon)$. Similarly,

$$
\int y^{\prime \prime} z^{\prime}\left(\eta Q_{2}\right)^{\prime}=\int y^{\prime \prime} z^{\prime} \eta^{\prime} Q_{2}+\int y^{\prime \prime} z^{\prime} \eta \eta^{\prime}\left(Q_{2}\right)_{\eta}+\int y^{\prime \prime} \eta z^{\prime 2}\left(Q_{2}\right)_{z},
$$

with $\left\|Q_{2}\right\|_{\infty},\left\|\left(Q_{2}\right)_{\eta}\right\|_{\infty}$, and $\left\|\left(Q_{2}\right)_{z}\right\|_{\infty}=\mathcal{O}(1)$.

We bound these terms as follows: First, note that by Inequality $A$, we have

$$
\int y^{\prime 2} \eta^{\prime 2} \leqq\left\|y^{\prime 2}\right\|_{2}\left\|\eta^{\prime 2}\right\|_{2} \leqq\|y\|_{\infty}\left\|y^{\prime \prime}\right\|_{2}\left\|\eta^{\prime 2}\right\|_{2} \leqq\|y\|_{\infty} \int\left(y^{\prime \prime 2}+\eta^{\prime 4}\right) .
$$

We next observe that

$$
\begin{aligned}
\int\left|y^{\prime \prime} z^{\prime \prime}\right| & \leqq \int y^{\prime \prime 2}+\int z^{\prime \prime 2} \\
\int\left|y^{\prime \prime} y^{\prime} \eta^{\prime}\right| & \leqq \varepsilon \int y^{\prime \prime 2}+\varepsilon^{-1}\|y\|_{\infty} \int\left(y^{\prime \prime 2}+\eta^{\prime 4}\right), \\
\int\left|y^{\prime \prime} y^{\prime} z^{\prime}\right| & \leqq \varepsilon \int y^{\prime \prime 2}+\varepsilon^{-1}\|y\|_{\infty} \int\left(y^{\prime \prime 2}+z^{\prime 4}\right), \\
\int\left|y^{\prime \prime} z^{\prime} \eta^{\prime}\right| & \leqq \varepsilon \int y^{\prime \prime 2}+\varepsilon^{-1}\left\|\eta^{\prime}\right\|_{\infty}^{2} \int z^{\prime 2}, \\
\int\left|y^{\prime \prime} z^{\prime 2}\right| & \leqq \varepsilon \int y^{\prime \prime 2}+\varepsilon^{-1} \int z^{\prime 4} .
\end{aligned}
$$


Applying again Inequalities $\mathrm{A}$ and $\mathrm{D}$, we have

$$
\left\|\eta^{\prime}\right\|_{\infty}^{2} \leqq\left\|\eta^{\prime}\right\|_{2}\left\|\eta^{\prime \prime}\right\|_{2}
$$

and

$$
\int z^{\prime 4} \leqq \mathcal{O}(1)\|z\|_{\infty}^{2} \int z^{\prime \prime 2}, \quad \int \eta^{\prime 4} \leqq \mathcal{O}(1)\|\eta\|_{\infty}^{2} \int \eta^{\prime \prime 2} .
$$

Using the bounds on $Q_{1}, Q_{2}$, we get from all of the above

$$
\dot{M}=\frac{1}{2} \int y^{\prime 2} \leqq-(1-\mathcal{O}(\varepsilon)) \int y^{\prime \prime 2}+\mathcal{O}(\varepsilon) \int\left(\eta^{\prime \prime 2}+z^{\prime 2}+z^{\prime \prime 2}\right) \text {. }
$$

We can now combine the bounds on $L, W, V, Z, M$, and we get

$$
\begin{aligned}
& \frac{1}{2} \frac{d}{d t} \int\left(z^{\prime 2}+\eta^{\prime 2}+z^{2}+z^{\prime \prime 2}+\eta^{\prime \prime 2}+y^{2}+y^{\prime 2}\right) \\
& \quad \leqq-(1-\mathcal{O}(\varepsilon)) \int\left(z^{\prime \prime 2}+3 z^{\prime 2}+\eta^{\prime \prime 2}+z^{2}+z^{\prime \prime \prime 2}+\eta^{\prime \prime \prime 2}+y^{\prime 2}+y^{\prime \prime 2}\right) .
\end{aligned}
$$

From this we can conclude, using (2.2):

Proposition 2.1. Assume the initial data $\xi$ satisfy $\xi \in \mathscr{B}_{\varepsilon}$ for sufficiently small $\varepsilon$. Let $T>0$ be the first time $t$ for which the inequality

$$
\sup _{x}|\zeta(t, x)| \leqq \varepsilon
$$

is violated. Then for all $t, 0 \leqq t \leqq T$ one has $\xi_{t} \in \mathscr{B}_{\varepsilon}$.

End of Proof of Theorem 1.1. To prove Theorem 1.1 it suffices to show that $T$, in Proposition 2.1 above is in fact infinite. Indeed, consider Eqs. (1.12), (1.13). By the Maximum Principle, [PW], we have $\sup |\zeta(t, x)| \leqq \varepsilon$, as long as $\left|\eta Q_{1}\right|<1$. But since $\left|\eta Q_{1}\right|<\mathcal{O}\left(\varepsilon^{2}\right)$, this is the case for all $t \geqq 0$. The proof is complete.

\section{Convergence}

In this section, we address the question of convergence of the solution to one of the stationary solutions of Eq. (1.1). Ideally, one would expect that convergence should take place in the following sense:

Conjecture 3.1. For every initial datum satisfying the Main Assumption with sufficiently small $\varepsilon>0$, there is a stationary solution

$$
u^{*}(x)=\sqrt{1-q^{* 2}} e^{i q^{*} x+i \theta^{*}},
$$

such that for every finite interval $I \subset \mathbf{R}$ one has

$$
\lim _{t \rightarrow \infty} \sup _{x \in I}\left|u(x, t)-u^{*}(x)\right|=0 .
$$

The convergence of the type of (3.2) holds true in the case when $q_{+}=q_{-}$in Eq. (1.2), and in fact convergence even takes place in suitable $L^{p}$ spaces [CEE].

The case of $q_{+} \neq q_{-}$is more complex. One cannot expect convergence of $u$ in $L^{p}$, because the values at infinity are different. However, one can study the convergence of the time derivative. If, for example, $|\dot{u}(x, t)| \leqq C(x) t^{-3 / 2}$, then clearly the pointwise time-limit $\lim _{t \rightarrow \infty} u(x, t)$ will exist. A situation where this really happens is 

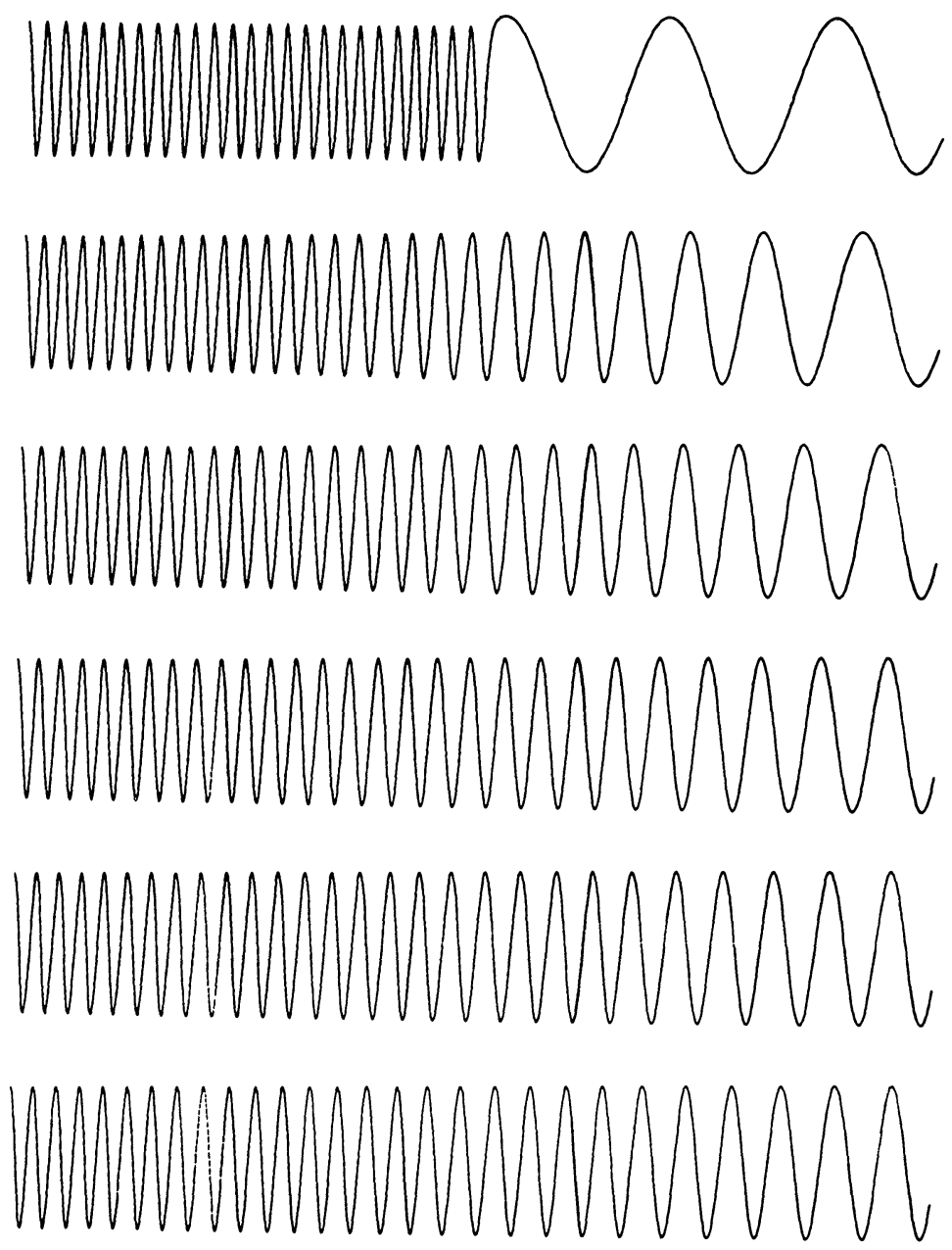

Fig. 1. Six frames of the evolution of the real part of $u$ for an initial condition with $q_{+} \neq q_{-}$. (The figure is produced with $\phi=\phi^{\prime \prime}$ and with $r^{2}+\phi^{\prime 2}=1$.)

encountered with the pure diffusion equation $\dot{\eta}=\eta^{\prime \prime}$, which is a caricature of the phase equation (1.4).

Indeed, it is easy to check that $\eta(x, t)=h(x / \sqrt{1+t})$ is a solution if $h^{\prime}(v)=A e^{-v^{2} / 2}$. Therefore, integrating the Gaussian from $v=0$, we see that $\eta$ equals an error function, and $\eta( \pm \infty, t)=B \pm \sqrt{2 \pi} A$, so that $\eta$ has different limits at $\pm \infty$. Clearly,

$$
\dot{\eta}(x, t)=-\frac{1}{2} h^{\prime}\left(\frac{x}{\sqrt{1+t}}\right) \frac{x}{(1+t)^{3 / 2}} .
$$

For fixed $x$ this is integrable in $t$, but it is clear that the convergence is not uniform in $x$. We believe that the same mechanism takes place in Eq. (1.1), and we illustrate this in Fig. 1 by replacing the phase equation (1.4) by the diffusion equation. The number of phase rotations in the space interval $[-L, L]$ at time $t$ is then 


$$
\int_{-L}^{L} d x \eta(x, t)=\sqrt{1+t} \int_{-L / \sqrt{1+t}}^{L / \sqrt{1+t}} d \xi h(\xi)=2 L\left(h(0)+\frac{L}{2 \sqrt{1+t}} h^{\prime}(0)+\mathcal{O}\left(\frac{L^{2}}{t}\right)\right) .
$$

Thus, the adaptation of the phase rotation is diffusive and consists in a rearrangement of phases at infinity. This can be done without phase slips, as is illustrated in Fig. 1.

At present, we are not able to prove the Conjecture 3.1, but only the following weaker statement:

Theorem 3.2. There is an $\varepsilon_{0}>0$ such that if the initial data satisfy the Main Assumption with $\varepsilon<\varepsilon_{0}$, then the solution converges in the following sense:

$$
\begin{aligned}
& \lim _{t \rightarrow \infty}\|z(\cdot, t)\|_{\infty}=0, \\
& \lim _{t \rightarrow \infty}\left\|z^{\prime}(\cdot, t)\right\|_{\infty}=0, \\
& \lim _{t \rightarrow \infty}\left\|\eta^{\prime}(\cdot, t)\right\|_{\infty}=0, \\
& \lim _{t \rightarrow \infty}\|y(\cdot, t)\|_{\infty}=0 .
\end{aligned}
$$

Remark 1. The convergence is on the derivatives only, and not on the functions themselves, because the values at infinity are unchanged in time for the function $\zeta$, and they are not 0 . Furthermore, as we have already mentioned in the introduction, the statement of Theorem 3.2 only implies convergence locally in time; Theorem 3.2 implies the existence of a function $\eta^{*}(t)$, (of $t$ only!) such that

$$
\lim _{t \rightarrow \infty} \sup _{x \in I}\left(|z(x, t)|+\left|\eta(x, t)-\eta^{*}(t)\right|+|y(x, t)|\right)=0 .
$$

At any given instant, the solution looks like one of the stationary solutions, but at some later time it can look like another stationary solution. Note that Eqs. (3.3) imply similar bounds on quantities more directly related to $u(x, t)$. For example, we have

$$
r^{\prime}=s^{\prime}=z^{\prime}+f_{z} z^{\prime}+f_{\eta} \eta^{\prime}
$$

so that $r^{\prime}$ tends to zero as well, since $f_{z}$ and $f_{\eta}$ are bounded.

Remark 2. Our difficulty in proving pointwise convergence is related to the function $\zeta$, cf. Eq. (1.12). Although we have bounds on the coefficient $\left(1+\eta Q_{1}\right)$, these bounds seem not good enough with respect to time convergence to guarantee the existence of pointwise limits for $\zeta(x, t)$ as $t \rightarrow \infty$. The literature contains very sharp Harnack inequalities, cf. [M, FS], but these only guarantee convergence to "flat" functions locally in time. But, such methods do not exclude a time evolution of those flat functions.

Proof. The proof will be seen to be an easy consequence of Theorem 1.1 and of Eq. (2.14). Let $Y$ denote the left-hand side of (2.14), and $U$ the right-hand side. Then $Y(t)$ is a bounded function of $t$, and hence each term in $Y$ is bounded as a function of $t$. If we reverse the inequalities and the signs in Eq. (2.14), then we get

$$
\left|\int_{t}^{t^{\prime}} d \tau U(\tau)\right| \leqq \frac{1}{2(1-\mathcal{O}(\varepsilon))}\left|Y(t)-Y\left(t^{\prime}\right)\right|=\mathcal{O}(\varepsilon)
$$


under the assumptions of Theorem 1.1. Therefore each of the terms in $U$ is integrable as a function of $t$. Since $Y(t)$ is bounded, this in turn implies

$$
\lim _{t \rightarrow \infty}|Y(t)-Y(\infty)|=0 \text {, }
$$

so that $Y$ has a limit as $t \rightarrow \infty$. In fact, from (2.6), (2.7), (2.9), (2.12), and (2.13), we see that

$$
\left\|z_{t}^{\prime}\right\|_{2}^{2}+\left\|\eta_{t}^{\prime}\right\|_{2}^{2},\left\|z_{t}\right\|_{2},\left\|z_{t}^{\prime \prime}\right\|_{2}^{2}+\left\|\eta_{t}^{\prime \prime}\right\|_{2}^{2},\left\|y_{t}\right\|_{2},\left\|y_{t}^{\prime}\right\|_{2}
$$

all have limits as $t \rightarrow \infty$. One can do better by inspecting the last two lines of Eq. (2.4), which show that $\left\|z_{t}^{\prime}\right\|_{2}$ and $\left\|\eta_{t}^{\prime}\right\|_{2}$ converge individually since, e.g., the bound on $z^{\prime}$ is

$$
\frac{d}{d t} \frac{1}{2} \int z^{\prime 2} \leqq-\int z^{\prime \prime 2}-2 A \int z^{\prime 2}+B,
$$

with $B$ an integrable function of time. Similarly, the calculations leading up to Eq. (2.9) show that $\left\|z_{t}^{\prime \prime}\right\|_{2}$ and $\left\|\eta_{t}^{\prime \prime}\right\|_{2}$ converge individually. Hence every integral on the left-hand side of Eq. (2.14) has a limit as $t \rightarrow \infty$.

Observe now that there are integrals occurring on both sides of (2.14). These terms are integrable (as functions of $t$ ) and have limits (as functions of $t$ ) and therefore these limits must be zero. Thus,

$$
\left\|z_{t}\right\|_{2}+\left\|z_{t}^{\prime}\right\|_{2}+\left\|z_{t}^{\prime \prime}\right\|_{2}+\left\|y_{t}^{\prime}\right\|_{2}+\left\|\eta_{t}^{\prime \prime}\right\|_{2} \rightarrow 0,
$$

as $t \rightarrow \infty$. Since we also have bounds on all the norms in (1.15), we can use the Schwarz inequality and get, e.g.,

$$
\left\|\eta_{t}^{\prime}\right\|_{\infty}^{2} \leqq 2 \int d x\left|\eta_{t}^{\prime} \eta_{t}^{\prime \prime}\right| \leqq\left\|\eta_{t}^{\prime}\right\|_{2}\left\|\eta_{t}^{\prime \prime}\right\|_{2} \rightarrow 0
$$

Using this device, we get

$$
\left\|z_{t}\right\|_{\infty}+\left\|z_{t}^{\prime}\right\|_{\infty}+\left\|y_{t}\right\|_{\infty}+\left\|\eta_{t}^{\prime}\right\|_{\infty} \rightarrow 0
$$

as $t \rightarrow \infty$. This completes the proof of Theorem 3.2.

Acknowledgements. We have profited from very helpful discussions with J. Moser concerning the Harnack inequalities. This work begun at IHES and was supported partially by DRET and the Fonds National Suisse.

\section{References}

[CE] Collet, P., Eckmann, J.-P.: Instabilities and fronts in extended systems: Princeton, NJ: Princeton University Press 1990

[CEE] Collet, P., Eckmann, J.-P., Epstein, H.: Diffusive repair for the Ginsburg-Landau equation. Helv. Phys. Acta (in press)

[FS] Fabes, E. B., Stroock, D. W.: A new proof of Moser's parabolic Harnack inequality using the old ideas of Nash. Arch. Rat. Mech. Anal. 96, 327-338 (1986)

[LA] Langer, J. S., Ambegaokar, V.: Intrinsic resistive transition in narrow superconducting channels. Phys. Rev. 168, 498-510 (1967)

[M] Moser, J.: On a pointwise estimate for parabolic differential equations. Commun. Pure and Appl. Math. 24, 727-740 (1971)

[PW] Protter, M., Weinberger, H.: Maximum principles in partial differential equations. Englewood Cliffs, N.J.: Prentice Hall 1967 